\title{
Reviewers for Volume 10
}

In addition to members of the editorial board, the following people served as consulting editors for Volume 10:

Patrick DeLeon, United States Senate

Howard Egeth, Johns Hopkins University

Nancy Eisenberg-Berg, Arizona State University

Valerie Hans, University of Delaware

Martin Kaplan, Northern Illinois University

Ingo Keilitz, National Center for State Courts

Michael Leippe, Adelphi University

Sally Lloyd-Bostock, Oxford University

Michael McCloskey, Johns Hopkins University

Trevor Nagel, Harvard University

Stephen Pfohl, Boston College

Ronald Roesch, Simon Fraser University

John Ryan, American Bar Foundation

Lita Linzer Schwartz, Pennsylvania State University

Charles Tremper, University of Nebraska

Walter Wadlington, University of Virginia

Carol Weiss, Harvard University

A. Daniel Yarmey, University of Guelph 